\title{
A New Kinetics and the Simplicity of Detonation
}

F.E. Walker

Interplay, Danville, California, U.S.A.

\section{ABSTRACT}

The results are presented from three experiments, as well as a number of molecular dynamics and quantum mechanics calculations, which cast in serious doubt the validity of some concepts and theories of detonation. This doubt led to numerous studies in search of more satisfying concepts, and the quite surprising results of several of those studies are given. Particularly, a new concept of the kinetics of shockinduced chemical reaction is presented. This process, designated as physical kinetics, is described as a nonequilibrium, nonthermal process in which chemical reaction rates are determined and regulated by the averaged vibrational velocities of the bonded atoms in condensed systems under the influence of high velocity shock waves. These velocities limit the advance of the kinetic energy which leads to the very high impact velocities of the atoms and molecules which cause massive bond fracture in the molecules in extremely short times. The majority of the free atoms and radicals and other highly activated species formed then react in very short times $\left(10^{-14}\right.$ to $\left.10^{-12} \mathrm{~s}\right)$, often in chain reactions, to provide the chemical energy which maintains the enormous level of kinetic energy at the detonation front. These high levels ensure that many reaction pathways are available--not only those with the lower activation energies or barrier potentials. It is in this regime of the detonation process that the more normal chemistry begins and then continues in other subsequent reactions to produce the adiabatic expansion forces and the final product mixtures. It is shown that this detonation model based on the new kinetics model, with the major initial reactions occurring in times of the order of tenths of picoseconds and in distances on the order of tens of angstroms--in the shock or detonation front-can provide a precise and satisfying mathematical and physical description of detonation phenomena. 


\section{INT RODUCTION}

There are a number of experiments and calculations from molecular dynamics, MD, and quantum mechanics, QM, which cast in serious doubt the validity of what have been called the classical concepts and theories of detonation. (Ref. 1)

Presented here are a new concept of kinetics (Ref. 2), applicable particularly to shock-induced chemical reactions, but also in many other very fast reactions, and a very different theory of detonation, with a simpler rationale that contributes to the confidence in and the utility of its application. The principal elementary physical principles used as the basis

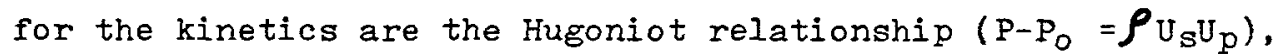
a concept of the momentum transfer of the shock energy, and a consideration of the efficacy of the kinetic energy of the atoms and molecules in a shock front in the mechanical fracture of covalent bonds in extremely short times. It will be shown that a detonation model based on this kinetics model, with the major initial reactions occurring in times of the order of tenths of picoseconds and in distances on the order of tens of angstroms-in the shock or detonation front, can provide a precise and satisfying mathematical and physical description of detonation phenomena.

Particularly, strong phenomenological evidence and data will be presented supporting the proposals that the kinetic energy from the shock forces, with the addition in a few tenths of a ps of a large fraction of the available chemical energy to the atoms in and near the front, can fracture serially a major portion of the covalent bonds of an explosive. The detonation velocity can now be calculated from the weight-averaged shock velocities of the component elements of the explosive. This method of calculation demonstrates that a minimal contribution to the detonation velocity is made by equilibrium thermal processes or thermodynamic factors, and that a key factor is simply the Hugoniot relationship (shock velocity versus pressure) of the elements of the empirical formulae of the explosives. 


\section{BACKGROUND}

The microscopic details of the chemical and physical phenomena by which a few grams of a seemingly quiescent and stable organic material can be converted in one or two microseconds into a mainly gaseous product, at temperatures of $2500-5000 \mathrm{~K}$ and pressures up to nearly 400,000 atmospheres, have eluded explosives scientists for more than a century. It will be shown herein that two very simple empirical equations and one other physically-meaningful equation can provide values of detonation velocities as well as or better than complex computer codes (Ref. 3), and that the new physical kinetics provides a rational explanation of the microscopic details of the phenomena.

If we review the data on 14 commonly-used explosives, we find they have, as measured and calculated, detonation pressures, $\mathrm{P}_{\mathrm{d}}$, covering a range of 13.0 to $39.0 \mathrm{GPa}$ and detonation velocities, $D$, from about 6.32 to $9.11 \mathrm{~km} / \mathrm{s}$. It is seen now that a three-fold increase in $P_{d}$ increased $D$ by only $44 \%$. It is important to consider also that a velocity of $9.11 \mathrm{~km} / \mathrm{s}$ is also 9.11 angstroms $/ 10^{-13} \mathrm{~s}$. This means that the atoms in the detonation front are being accelerated by momentum transfer on a ps time scale to energies near $5 \mathrm{eV}$, and the front is crossing the covalent bonds of these organic compounds in one to a few vibration periods. This indicates that there is sufficient energy to break most of the bonds in the explosives molecules to provide many reaction pathway options, * not simply the ones with the lowest energy barriers or activation energies, and that this fracture can occur in time scales near 10-14 $\mathrm{s}$. (Ref. 3)

Experiments and molecular dynamics calculations (Ref. 1) give strong evidence that the width of the shock front is on the order of 50 angstroms, with a rise time in the ps range.

* This consideration also provides a rationale for low velocity detonation in an explosive initiated at a lower shock pressure, as well as for the increase in $D$ for nitromethane with diethylenetriamine. It is feasible that a stable reaction regime could be established with less massive bond fracture, leading to a lower level of chemical reaction in and near the detonation front and thus a lower $P_{d}$ and detonation velocity. 
The MD studies further show that a major (about $80 \%$ ) part of the energy of momentum transfer in the front, which begins to flow into the vibrational component in the molecular bonds in a few femtoseconds, has risen to levels near $4 \mathrm{eV}$ in about $80 \mathrm{fs}$. MD studies also show that this violent energy fluence or flux (about $5 \mathrm{ev} / \mathrm{ps}$ ) causes scission of covalent bonds by impact, compression or shear forces to produce very energetic free atoms and radicals in excited states. (Ref. $1,2,3$ )

In a momentum transfer involving an $N$ atom at $8 \mathrm{~km} / \mathrm{s}$ with $4.72 \mathrm{eV}$ of kinetic energy to a surface $\mathrm{N}-\mathrm{N}$ couple with a bond strength of $1.06 \mathrm{eV}$, there is a high probability the bond would be broken and the exterior $\mathrm{N}$ atom would be given translational energy of about $3.5 \mathrm{eV}$ and a velocity of $6.5 \mathrm{~km} / \mathrm{s}$. (Ref. 3)

Three of the reasons it is important to have a new detonation theory are now discussed. (See Ref. 1) We completed a series of experiments on the low-pressure ( 5.1 to $6.5 \mathrm{GPa}$ ) initiation of nitrometnane, NM, (Ref. 4) and found (1) that the time to initiation was about 4 orders of magnitude shorter than predicted by the thermodynamic theory, and also (2) the pattern of the initiation process was much different from the classical model. (Ref. 4) We also carried out a series of initiation experiments in which diethylenetriamine, DETA, was added to NM. (Ref. 5) one observation from this series was (Reason 3) that the detonation velocity of the NM was increased to about 6.72 $\mathrm{km} / \mathrm{s}$ from the nominal measured value of $6.32 \mathrm{~km} / \mathrm{s}$ for neat $\mathrm{NM}$, by the addition of only $0.05 \%$ of DETA. This would not be explainable with the thermodynamic concepts, but it is easily defended under the new theory presented herein. In fact, we proposed that the DETA would provide $\mathrm{NH}$ radicals and free $\mathrm{N}$ and $\mathrm{H}$ atoms which could provide new chain reaction pathways to increase the energy release rate. A calculation using the skidmore-Hart equation (Ref. 1) for overdriven detonations showed a probable very high $P_{d}$ of about $19 \mathrm{GPa}$ had been attained. Using the new equation given later (based only on the elemental Hugoniots) for calculating $D^{\prime} \mathrm{s}$, we see that the new $D$ should have been near $6.7 \mathrm{~km} / \mathrm{s}$ for the NM with $0.05 \%$ DETA, as we measured. (Ref. 1) 


\section{DEFINITIONS}

Physical Kinetics. In detonations and some other very fast and shock-induced reactions, the rates of reaction are determined by the physical limitations of the advance of the activating energy of bond fracture through the reacting materials. This is essentially a nonthermal, nonequilibrium process related to the shock velocities, $U_{S}$, of the individual elements of the reactive materials.

Detonation. The new detonation theory, based on the concept of physical kinetics, includes the experimental and calculational observations that nearly all of the covalent bonds of the explosives molecules are broken or rearranged within the detonation shock front (about 20-100 angstroms) by impact, compression and shear forces, and that the majority of the free atoms and radicals and other highly activated species formed then react in very short times $\left(10^{-14}\right.$ to $\left.10^{-12} \mathrm{~s}\right)$ to release chemical energy which maintains the enormous levels of kinetic energy at the detonation front. Other subsequent more normal reactions provide the adiabatic expansion forces and the final product mixtures. since the molecules are essentially broken down to their elements in the shock front, the detonation velocities are determined by the weight-averaged shock velocities of the elements of the empirical formulae. It follows that thermodynamics has only a secondary role, and there is probably insufficient time in this initial phase for the anharmonic coupling of excited phonon modes with the low frequency molecular vibrations.

NEW CONSIDERATIONS OF SHOCK VELOCITY

The basic equation for shock velocity calculations is the Hugoniot relationship, $P-P_{O}=\rho_{U_{s}} U_{p}$, where $P$ is shock pressure, $\rho_{i s}$ density, and $U_{s}$ and $U_{p}$ are shock and particle velocity, respectively. However, it appeared, from some information from MD calculations, that one might be able to calculate shock velocities of the condensed elements from simply the data in the periodic chart--atomic weight, atomic radius and density. Studies in this regard led to the equation, 


$$
U_{\mathrm{S}}=\left(\frac{\mathrm{r}_{\mathrm{a}}}{\mathrm{w}_{\mathrm{a}}}\right)^{\frac{1}{4}} \rho-0.1 \mathrm{f}(\mathrm{P})
$$

where $f(P)=\left(0.42 P+10.3 P^{\frac{1}{2}}+12\right)$ for most of the elements, and only a slightly modified $f(P)$ gives excellent results for those elements with relatively large atomic radii. The attainable accuracy is shown in Fig. 1 (Ref. 6) This accuracy corresponds well with the precision of the data.

The next step in this Iine of investigation was to determine if the Hugoniots of organic compounds could be calculated from the $U_{S}$ versus $P$ information calculated for the elements. Again, a quite simple equation was derived,

$$
u_{s c}=\sum\left(u_{s i} f_{i}\right)
$$

in which $U_{s c}$ is the shock velocity of the compound, the Usi's are the shock velocities of the elements of the compound at a given $P$, and the $f_{i}$ 's are the weight fractions of the elements obtained from the empirical formulae of the compounds examined. (Ref. 7) This equation also gives very good results.

A Hugoniot "experiment" was conducted with a series of MD calculations in which the velocity of an impacting plate was increased in increments, and the resultant shock velocities in a representative covalently-bonded lattice were measured. (Ref. 1) The shock velocity values obtained, using two covalent potentials spanning the normal range found in organic compounds, are given in Fig. 2. Also given are the measured shock velocities for a number of organic plastics and explosives. It can be seen that the MD calculations compare favorably with the data. This fact adds confidence to the MD study of shock processes.

It was determined that the average relative vibrational velocities, $A R V V^{\prime} s$, of the covalent atomic pairs (C-H, N-H, O-H, $\mathrm{C}-\mathrm{N}$, etc.) could be calculated from thermal motion measureable in $x$-ray crystallographic data and, also, from the infrared spectrographic frequency data for specific bonds. (Ref. 8) A somewhat surprising and interesting correlation of these results with other shock phenomena is given in Fig. 3, where it is seen that all of the detonation velocities lie in the cross-hatched 
area which includes the shock velocities and the ARVV's of the $\mathrm{C}-\mathrm{H}, \mathrm{O}-\mathrm{H}$, and $\mathrm{N}-\mathrm{H}$ couples. (Ref. 9)

To obtain more precise values of the ARVV's, a series of QM calculations of the values for 10 selected atom pairs found in organic explosives was calculated using the least-squares fit of the diatomic potentials to Hulbert-Hirschfelder functions. (Ref. 10) The plot of these values versus energy levels for the 10 atom pairs is given as Fig. 4. The calculations show values of the ARVV's comparable to those calculated from the infrared and $x$-ray crystallographic data. These values all correspond closely to the shock velocities we calculated for the $\mathrm{C}, \mathrm{N}, \mathrm{O}$ elements and pairs with $H$ and for the organic compounds examined. These considerations will be shown to be key factors in the new kinetics and detonation concepts.

\section{KINETICS DISCUSSION}

In 1975, Henry Eyring (Ref. 11) showed that the ordinary concepts of chemical kinetics must be modified significantly to explain observed reaction rates in some shocked hydrocarbons and explosives. He proposed a concept which he designated as "starvation kinetics" to help explain why the high temperature (more than $1200 \mathrm{~K}$ ) decomposition of the different materials in his studies had nearly the same reaction rate, even though the low temperature (less than $500 \mathrm{~K}$ ) rates were rational, different from each other, and described quite well by Arrhenius principles. If the decomposition is assumed to obey first-order kinetics, then the logarithms of the rate constants at high temperature for all the materials Eyring studied were nearly equal and in the rather narrow range of 5.5 to 6.5 .

Even when one makes the obvious comments that first-order kinetics is probably not the only order involved and that both decomposition mechanisms and rates probably would change significantly over such a wide range of temperatures, this should in no way lead to the conclusion that all of the high temperature rates presented (see Fig. 5) should be nearly equal nor should they be approximately equal to first-order Arrhenius rate constant 
logarithms of 5.5 to 6.5. However, in the concept of physical kinetics, and with reference to the observations and calculations of shock velocities and the ARV''s, these rates should be about equal, and they should coincide with pseudo-first-order Arrhenius rates, as measured and observed. The rates for the detonation reactions fall in this range. (Ref. 9)

The alternative kinetics concept presented herein is that there is a physical regulator of the rate of transfer of the decomposition energy (principally, the atomic vibrational energy) from one molecule to the adjoining molecules or from one vibrating bond to others in its immediate vicinity. This regulator is the effective ARV''s of the vibrating atoms in the material while it is under shock loading. This nonthermal, nonequilibrium reaction kinetics, regulated by this energy transfer process, is designated as physical kinetics.

Early indicators for the requirement for a new kinetics hypothesis were these two observations: (1) Shock and detonation waves are moving past the atoms in condensed materials on the same time scales as the vibrational frequencies of the organic bonds; and (2) there is enough energy in a moderate shock front (about $7 \mathrm{GPa}$ ) to mechanically fracture a $\mathrm{C}-\mathrm{N}$ or $\mathrm{N}-\mathrm{O}$ bond in a representative chemical explosive, $\mathrm{RDX}$ (hexahydro-1,3,5-trinitro1.3.5-triazine). (Refs 3,12) The nominal energy of a $\mathrm{C}-\mathrm{N}$ bond in $\mathrm{RDX}$ is 0.37 aJ $\left(10^{-18} \mathrm{~J}\right)$, and for an $\mathrm{N}-\mathrm{N}$ bond it is $0.17 \mathrm{aJ}$. The kinetic energy of an 0 atom or an $N$ atom moving at $8 \mathrm{~km} / \mathrm{s}$ would be 0.86 aJ or 0.76 aJ, respectively. Thus, through momemtum transfer the impact of an 0 or an $N$ atom of these energies on an exterior $\mathrm{C}-\mathrm{N}$ or $\mathrm{N}-\mathrm{N}$ couple could mechanically fracture the bond. (Refs. 3,13)

The vibrational velocities of the atoms in an organic molecule are of the same velocity scales $\left(\mathrm{km} / \mathrm{s}\right.$ or angstroms $\left./ 10^{-13} \mathrm{~s}\right)$ as shock and detonation velocities. They can be calculated with a simple equation from infrared spectroscopic data, $v=\nu_{c u}$, where $V$ is the vibrational velocity, $\nu$ is the infrared-derived vibrational frequency in $\mathrm{cm}^{-1}$ of a specific bond (i.e., $\mathrm{C}-\mathrm{H}, \mathrm{N}-\mathrm{H}$, 
$0-H)$, $c$ is the velocity of light, and $u$ is the nominal distance relative to each other the specific vibrating atoms move in one vibration.

A key argument in support of physical kinetics and the importance of shock velocities in determining detonation velocities is given in Fig. 6. (Refs. 2,13) Here the averaged Hugoniot measurements for a number of organic compounds and the elements $C, 0$, and $N$ are plotted in comparison with the unreacted Hugoniot for TATB (s-triaminotrinitrobenzene), $\rho=1.876 \mathrm{~g} / \mathrm{cm}^{3}$. These two curves are very nearly congruent. Also plotted in Fig. 6 are the $D^{\prime} s$ and $P_{d}{ }^{\prime} s$ of 15 common, but both chemically and energetically diverse, condensed explosives.

What is clearly evident is that the detonating explosives have velocities at their detonation pressures only slightly higher than the shock velocities of the inert materials. A curve representing a $10 \%$ increase to the shock velocities of the nonexplosive elements and compounds was added to the graph to compensate for the higher temperature in detonation, and all of the explosives, except NM which is only slightly outside, are included within this parameter. (Refs. 2,13) Thus, it appears that any thermodynamic factors can have only a minimal effect in determining detonation velocities.

The chemistry immediately following the initial bond fracture (about $10^{-13}$ to $10^{-12} \mathrm{~s}$ ) is extremely important. By adding sources of new free radicals and thus new reaction chains, the rate of energy release can be increased, and higher pressures can be attained. This can lead to higher detonation velocities and to smaller critical diameters, as seen in the NM-DETA experiments. It has been demonstrated that very low levels of additive (less than 0.1\%) have large effects. (Refs. 5,13,14,15)

The addition of $0.08 \%$ DETA to NM-acetone mixtures yields a decrease of $80 \%$ of the mean cell size in the detonation. Addition of $0.1 \%$ DETA increased the acceptable dilution for detonation by $35.7 \%$. Finally, $0.03 \%$ of DETA in NM reduced the critical diameter by $43 \%$. (Ref. 13) These data support our observed increase in the D of NM with $0.05 \%$ added DETA. 


\section{MASSIVE BOND FRACTURE}

The BTNEA Experiment. A homogeneous ideal explosive, bistrinitroethyl adipate (BTNEA) was synthesized with isotopic labels ( $\mathrm{C}^{13}$ and $\mathrm{o}^{18}$ ) introduced into the positions indicated in Fig. ?. This explosive was chosen for this experiment, because it appeared that the $\mathrm{CO}$ and $\mathrm{CO}_{2}$ molecules expected as detonation products were already formed, and the isotopic labels would be found in the $\mathrm{CO}$ and $\mathrm{CO}_{2}$ products.

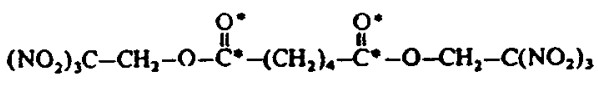

BTNEA

Figure 7. The structure of bis-trinitroethyl adipate. The asterisks indicate carbon atoms of isotope 13 and oxygen atoms of isotope 18 .

The explosive was detonated in a bomb calorimeter in which the products were collected and then analyzed for the isotopic ratios. (Ref. 16) The experimental results, in Table 1, show that the ratios of $\mathrm{C}^{12} / \mathrm{C}^{13}$ and $0^{16} / 0^{18}$ are essentially the same for all of the product species containing $C$ and/or 0 , and they are nearly equal to the isotopic ratios in the initial BTNEA sample. The analytical values of the ratios were said to be well within the experimental error of the determination. The conclusion that is obvious is that almost every covalent bond was broken, the atoms were scrambled, and they were randomly combined into the detonation products. Quoting from the paper, "We must conclude that, in the case of the homogeneous ideal explosive, all of the bonds of the original explosive molecule are, in effect, broken during the detonation process. These molecular fragments then must recombine in a statistically random fashion prior to the kinetic "freeze out" of products during the adiabatic expansion. Certainly, diffusion on a molecular level cannot be an important rate controlling process." (Ref. 16)

Comparative results of massive fracture of covalent bonds in and near a shock front in simulated organic matrices have been observed in the MD calculations of many workers and in our studies. (See Refs. 1,9,10,17,18-21) In many other experimental 
studies the mechanical scission of chemical bonds has been observed or proposed (Refs. 2,10,22-25), and it has been demonstrated in numerous shock-induced chemistry experiments. (Ref. 22) (See Figs. 8a,8b)

Data and information reported from reaction dynamics experiments appear to correlate very well with data and calculations from the study of detonation. The development of femtosecond lasers and their combination in experimental systems with the molecular beam technique, for monitoring energy states immediately before and after a chemical reaction, have provided an effective method for observing the transient state, TS, in a reaction as it forms and divides into products. (Refs. 26-28)

The results of reaction dymamics, $R D$, studies of a system in which a van der Waals molecule, IH・Ooco, undergoes UV photolysis which accelerates the $\mathrm{H}$ atom to about $20 \mathrm{~km} / \mathrm{s}$ toward the oco molecule, thus forming the TS, show the appearance of an $\mathrm{OH}$ signal in about 5 to 15 picoseconds after the deconvolution of the TS. In another experiment involving the decomposition of ICN, it was reported that the Ts has a lifetime of about 200 fs and a translational velocity of about $2 \mathrm{~km} / \mathrm{s}$. This shows that this TS exists for about four vibrations of the IC-N bond, and that the ICN molecule rotates about only $7^{\circ}$ during this period. The energy reported to be available for this reaction is about $0.87 \mathrm{eV}$, or near $7000 \mathrm{~cm}^{-1}$. (Refs. 27,28)

This experimentally-derived information appears to be directly related to data and calculations (MD and $Q M$ ) seen in the study of detonation (and initiation) of chemical explosives. There appear to be many fundamental correlations in these two chemical physics regimes-molecular and atomic velocities in $\mathrm{km} / \mathrm{s}$ and reaction times in the ps range. (See Ref. 3)

\section{CALCULATION OF DETONATION VELOCITIES}

From the foregoing data and discussion we can now present the perceived simplicity of this theory of detonation. For more than 100 years scientists around the world have struggled to obtain or derive equations, computer programs, sophisticated 
theories, and erudite and exotic equations-of-state, Eos, to use to calculate accurately detonation velocities. This has been accomplished, but in an often complex and complicated manner, with large computer codes.

Following are three amazingly simple equations that are used successfully to calculate detonation velocities with a high degree of accuracy. (Refs. 1,7,29) The results from the second and third show correlation coefficients with large sets of data of 0.971 and 0.954 , respectively. For sets involving the explosives which are best characterized, the coefficients are 0.991 and 0.976 .

$$
\begin{aligned}
& D=2.45+\frac{\mathrm{P}^{\frac{1}{2}}}{3} \\
& \mathrm{D}=\left(\frac{\mathrm{P}^{\frac{1}{2}}}{3}+2.0+0.25 \mathrm{a}\right)+0.05\left(\mathrm{H}_{\mathrm{p}}+\frac{\mathrm{N}_{\mathrm{p}}}{20}\right) \\
& \mathrm{D}=\mathrm{T}_{\mathrm{C}}\left[\left(\mathrm{U}_{\mathrm{S}_{\mathrm{i}}} \mathrm{f}_{i}\right)\right.
\end{aligned}
$$

Eq. 3 was developed algebraically from two empirical equations derived before 1969 to calculate detonation pressures and velocities. (Refs. 1,30) The use of this very elementary equation provided some evidence that detonation velocity was a rather weak function of pressure and that detonation was probably a much less complicated process than had been believed. Eq, 4 was developed from observations of the results from Eq. 3 . Specifically, it was easily seen that the aromatic molecules had about $0.25 \mathrm{~km} / \mathrm{s}$ lower $D^{\prime} \mathrm{s}$ for given $\mathrm{Pd}_{\mathrm{d}}{ }^{\prime} \mathrm{s}$, which could reflect the additional energy required to break up the aromatic rings and some of the more complex molecular structures. Additionally, compounds with relatively higher $\mathrm{H}$ and $\mathrm{N}$ content appeared to have slightly higher D's.

Calculations with Eq. 4 showed excellent correlations to the data. This observation, along with the new concepts of physical kinetics--that shock velocities and the ARVV's were related and similar (as proposed herein) and that massive kinetic fracture of the covalent bonds of an explosive in the shock front was probable, led to the development of Eq. 5. (Ref. 29) Here, 
$T_{c}$ is a small (about 2 to $6 \%$ ) correction to the shock velocity based on the fact that the temperatures of detonation are much higher than those at which Hugoniot values are usually measured. The correction curve is taken from the Hulbert-Hirschfelder calculations of $U_{S}$ versus $T$. (Ref. 29) The $U_{S i}$ and $f_{i}$ functions are simply the shock velocities of the elements of the empirical formula of an explosive at $\mathrm{Pd}$ and the weight fraction of each element, respectively. The excellent correlation to the data obtained with Eq. 5 (See Table 2) is a validation of the concepts presented earlier in this paper, which are summarized below:

1. Physical kinetics applies in determining $D . *$

2. Shock velocities of the component elements are key factors.

3. The kinetic energy in the detonation front leads to massive fracture of the covalent bonds in and near the front, by impact, compression and shear.

Thus, thermodynamics, excited atomic and molecular states, the transfer of energy from shock produced phonons to the internal vibrations of the molecules, electronic transitions, and some other often considered factors, although certainly involved at some level, have a relatively minor influence on detonation velocity.

If the molecules were not broken into their component atoms at or very near the front, Eq. 5 probably would not represent a rational concept, and it is highly improbable that it would provide any correct calculations of D--certainly not a set of 4 ? with a correlation coefficient of 0.954 .

*There may be some intrinsic regulation of detonation velocities involving velocities of impact of atomic and molecular species, orientation of impacted molecules or bonded couples, and resonance relationships between impact velocity and vibrational frequency of impacted molecular bonds, but these factors do not appear to be required considerations for the detonation velocity determination. 
Additionally, in a more recent report (Ref. 31), an explosive designated as $\mathbf{2} 25$ ( $75 \%$ PETN/25\% paraffin) at a density of $1.265 \mathrm{~g} / \mathrm{cm}^{3}$ has a measured $D$ of $7.230 \mathrm{~km} / \mathrm{s}$, whereas pure PETN (pentaerythritol tetranitrate) at the same density has a measured $D$ of $6.60 \mathrm{~km} / \mathrm{s}$. Using the thermodynamic codes, E25 showed a calculated D of $6.20 \mathrm{~km} / \mathrm{s}$. However, the calculation with Eq. 5 gave a value of $7.267 \mathrm{~km} / \mathrm{s}$, within $0.51 \%$ of the measured value. This is well within the precision of $\mathrm{D}$ measurements. The classical theory calculation missed the measured value by $-14.24 \%$. The author of the paper who reported the E25 data (Ref. 31) stated that, "All equations-of-state available to us cannot reproduce these results." This relatively recent observation is compelling support for the concepts described herein.

ADDITIONAI CONSIDERATIONS

Analysis of a set of data onithe shock initiation of PBX-9404 (an HMX-based, plastic-bonded explosive) led to the derivation of the critical energy fluence equation which provides the criteria for the shock initiation of explosives. This equation is:

$$
E_{c}=\frac{t P^{2}}{\rho U_{s}}
$$

where $t$ is the time-width of an initiating shock of velocity $U_{S}$ providing a pressure $P$ in the shocked explosive. The initial density of the explosive is $\rho$. The equation is derived from simple basic physics equations involving kinetic energy and shock velocity, showing the importance of those factors in initiation as well as in detonation. This critical energy equation has been used successfully for about two decades in numerous shock initiation studies (Refs. 32,33), for designing explosives-activated escape systems for aerospace applications, and for many other purposes. (Ref. 34)

Another interesting factor appears in our work and the work of A.N. Dremin. (Refs. 9,35) The one-dimensional translational "temperature" of the atoms in the shock front of a nominal $5 \mathrm{GPa}$ shock is calculated to be greater that $12,000 \mathrm{~K}$, and for $\mathrm{N}$ and 0 
atoms, accelerated by momentum transfer in the detonation front to $8 \mathrm{~km} / \mathrm{s}$, these translational pseudo-temperatures would be about $20,000 \mathrm{~K}$ or higher.

For many years, since at least 1974, we have maintained that the very high levels of kinetic energy in the detonation front provide levels of impact, compression, and shear forces sufficient to cause mechanical fracture of a major portion of the covalent bonds. (Refs. $1,2,3,12,36,37$ ) Much recent work in molecular mechanics, particularly by J. Gilman (Ref. 39) provides a quantitative mathematical and chemical description of the mechanical (nonthermal) bond fracture mechanisms, which may apply in shock-induced reactions and detonation.

We have proposed that the chemistry immediately following the initial bond fracture (about $10^{-13}$ to $10^{-12} \mathrm{~s}$ ) is extremely important. By adding sources of new free radicals, and thus new reaction chains, the rates of energy release could be increased and higher reaction pressures could be attained. This can lead to higher detonation velocities and to smaller critical diameters, as has been observed in many NM-DETA experiments. (Refs. 5,14, 15.36) These additional considerations add credence to our experimental observations and calculations.

\section{CONCLUSIONS}

It is concluded: (1) That the new concept of physical kinetics is a valid concept for determining reaction rates in detonations and in highly shocked systems, and that the methods given for the calculation of shock velocities for the elements and compounds and explosives mixtures are based on proper physical principles. These shock velocities are related directly to the ARVV's and to detonation velocities.

(2) That the exceedingly high kinetic energy in the detonation front is sufficient to cause massive fracture of the covalent bonds of the molecules of the explosives at and near the front, so that the large majority of the molecules are broken to individual atoms or radicals and rearranged extensively, and that the subsequent very rapid chemistry can be influenced by the 
addition in the explosives of chemicals providing enhancing or inhibiting reactions or other chemicals which could influence sensitivity.

(3) That the simple equation, $D=T_{c} \sum\left(U_{s i} f_{i}\right)$, is a rational equation, based on appropriate Hugoniot principles, which provides for the very accurate calculation of detonation velocities from the shock velocities of the elements in the empirical formulae of the explosives.

\section{ACKNOWLEDGMENTS}

I wish to acknowledge helpful conversations regarding this paper with R.J. Wasley, A.N. Dremin, H.N. Presles, S. Odiot, J.J. Gilman, and D.D. Dlott.

TABLE 1. ISOTOPIC RATIOS IN BTNEA AND ITS DETONATION PRODUCTS

\begin{tabular}{cccc} 
& & $\mathrm{C}^{12} / \mathrm{C}^{13}$ & $0^{16} / \mathrm{0}^{18}$ \\
\hline $\begin{array}{c}\text { Labeled BTNEA } \\
\text { Products }\end{array}$ & & 4.8 & 11.7 \\
& $\mathrm{H}_{2} \mathrm{O}$ & - & 16.6 \\
& $\mathrm{CO}_{2}$ & 4.7 & 11.4 \\
& $\mathrm{CO}^{2}$ & 4.8 & 11.2 \\
& $\mathrm{CH}_{4}$ & 4.5 & - \\
& $\mathrm{C}$ & 4.6 & - \\
\hline
\end{tabular}

TABLE 2. DETONATION VELOCITIES CALCULATED FROM EQUATION 5

\begin{tabular}{|c|c|c|c|c|c|}
\hline Explosive* & $\begin{array}{l}\text { DATA } \\
\mathrm{P}(\mathrm{GPa})\end{array}$ & $D(\mathrm{~km} / \mathrm{s})$ & $\begin{array}{l}\text { CALCULAT } \\
D_{C}(\mathrm{~km} / \mathrm{s})\end{array}$ & $\mathrm{D}$ & $\%$ Dev. \\
\hline $\begin{array}{l}\text { BTF } \\
\text { DATB } \\
\text { HMX } \\
\text { HNS } \\
\text { PETN } \\
\text { RDX } \\
\text { TATB } \\
\text { Tetryl } \\
\text { TNT }\end{array}$ & $\begin{array}{l}36.0 \\
25.9 \\
39.0 \\
20.0 \\
33.5 \\
33.8 \\
29.1 \\
26.0 \\
21.0\end{array}$ & $\begin{array}{l}8.49 \\
7.52 \\
9.11 \\
6.80 \\
8.26 \\
8.70 \\
7.87 \\
7.50 \\
6.94\end{array}$ & $\begin{array}{l}8.50 \\
7.62 \\
9.09 \\
6.83 \\
8.21 \\
8.59 \\
7.98 \\
7.55 \\
7.01\end{array}$ & $\begin{array}{l}0.01+ \\
0.10+ \\
0.02- \\
0.03+ \\
0.05- \\
0.11- \\
0.11+ \\
0.05+ \\
0.07+\end{array}$ & $\begin{array}{l}0.12+ \\
1.33+ \\
0.22- \\
0.44+ \\
0.61- \\
1.26- \\
1.40+ \\
0.67+ \\
1.01+\end{array}$ \\
\hline
\end{tabular}




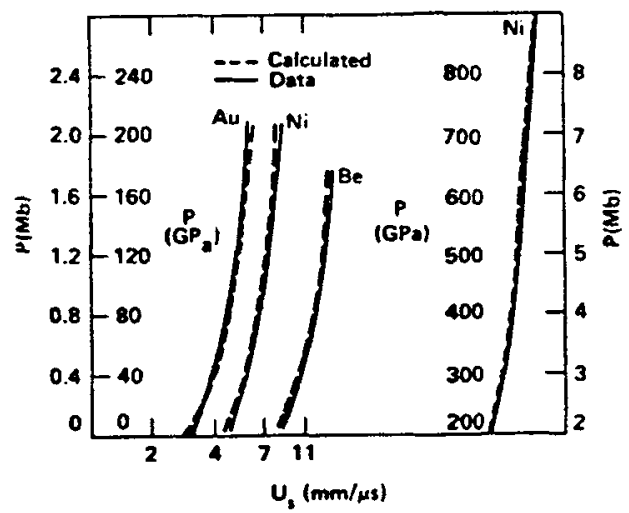

Figure 1. US versus $P$ curves for beryllium, nickel, and gold calculated with Equation (1).

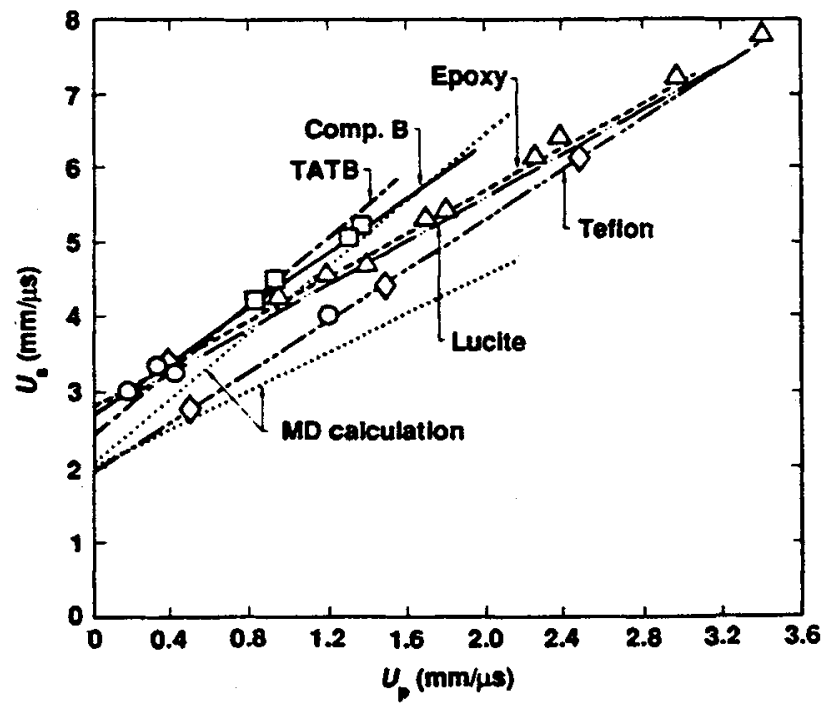

Figure 2. The Hugoniot plot of common organic plastics and explosives compared with the MD Hugoniot calculations. 

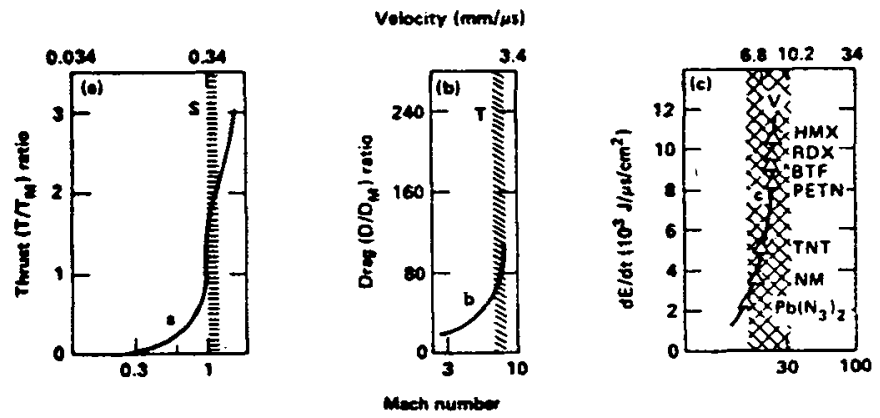

Figure 3. Representation of sonic (S), thermal (T) and shock (V) "barriers" as related to (a) sonic flow, (b) hypersonic flow, and (c) detonation velocity.

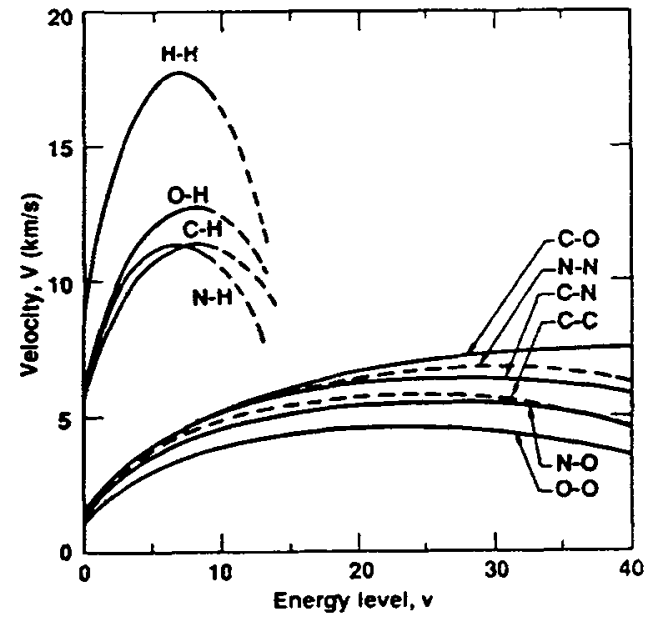

Figure 4. Plot of average vibrational velocity vs energy level for the ten atom pairs considered here. 


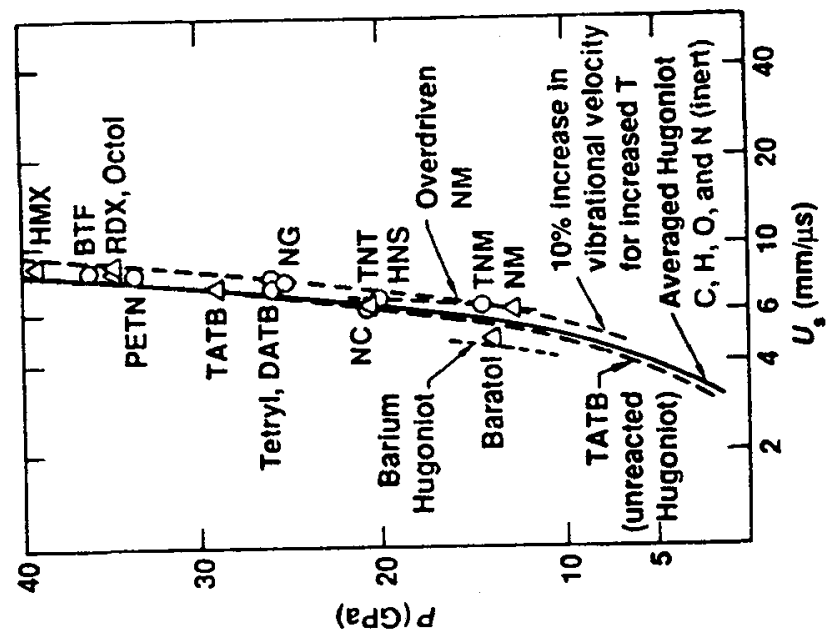
落

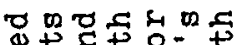

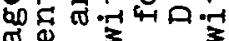
近的 0

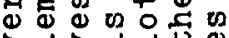
उ

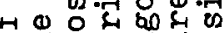

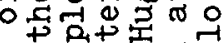

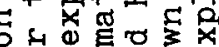
응 吸要

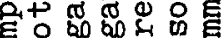

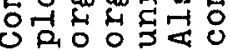

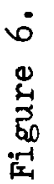

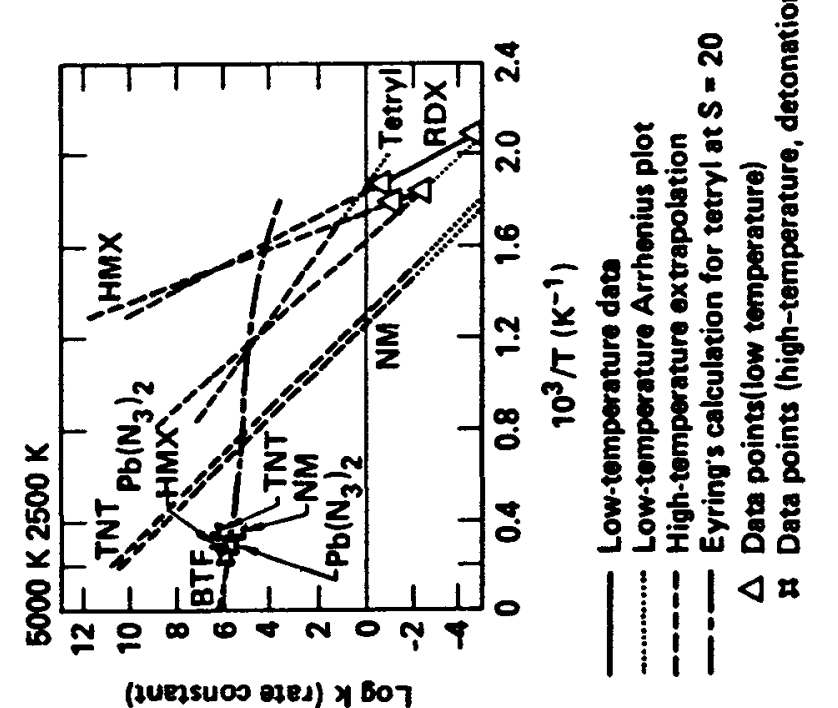

40. 옳 †点昆

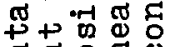
ه 吕罂。 ⿷匚

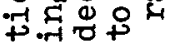

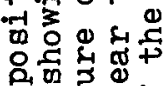
है क نू

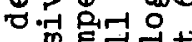
ᄀ웡 15

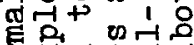
E्र (1) in 


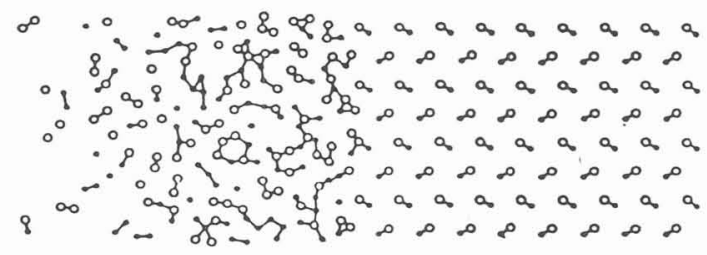

Snapshot from a sitnulation of a detonating film. The length of the systern slowwil is $\approx 80 \AA$.

Figure 8a. Molecular dynamics calculation of a model detonating solid with two types of atoms and with exothermic reactivity incorporated into the dynamics.

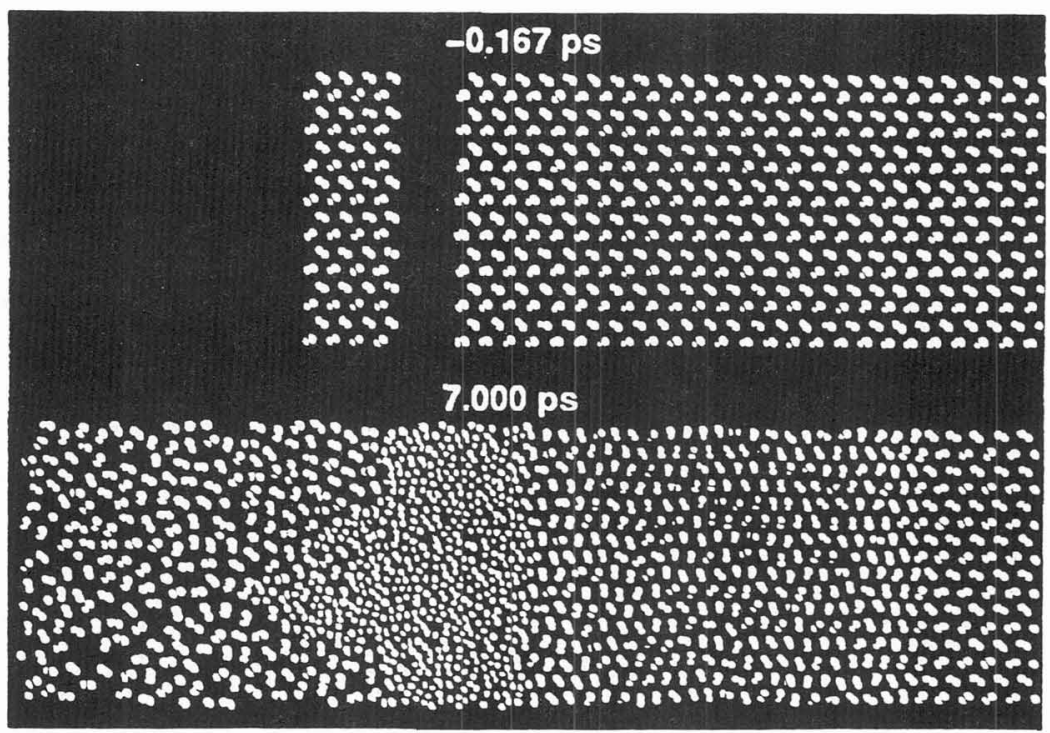

Figure 8b. Molecular dynamics calculation with Tersoff-like potentials used to simulate the detonation of an energetic two dimensional semi-infinite molecular solid. 


\section{REFERENCES}

1. F.E. Walker, Lawrence Iivermore National Iaboratory, CA Report No. UCRL-53860, 11 January 1988.

2. F.E. Walker, J. Appl. Phys. 63, 5548 (1988).

3. F.E. Walker, Propel1., Explo., Pyrotech. 15, 190 (1990).

4. F.E. Walker and R.J. Wasley, Combustion and Flame 15 , 233 (1970).

5. F.E. Walker, Acta Astronautica 6, 807, Pergamon Press Itd, (1979).

6. F.E. Walker, F.G. Walker and J.B. Walker, J. Appl. Phys. $60,3876(1986)$.

7. F.E. Walker, Evidence of a Physical and Kinetics Basis for Detonation Velocity and Stability, Naval Research Lab., Tech. Report, 17 August 1988.

8. A.M. Karo and F.E. Walker, Proceedings of the APS 1981 Topical Conference on Shock Waves in Condensed Matter, Menlo Park, CA, 23-25 June 1981.

9. F.E. Walker, Propellants and Explosives 6, 15 (1981).

10. F.E. Walker and A.M. Karo, Shock Waves in Condensed Matter 1987. S.C. Schmidt, N.C. Holmes (editors) Elsevier Science Publishers B.V., p. 543 (1988).

11. H. Eyring, Chem. Eng. News 53, 27 (1975).

12. F.E. Walker, Lawrence Iivermore National Laboratory, CA Report No. UCRL-75722, Rev. 1, 21 April 1975.

13. F.E. Walker, Proceedings of the Intemational Conference on High Energy Rate Fabrication (HERF), Ijubljana, Slovenia, 18-22 September 1989.

14. H.N. Presles and D. Desbordes, Revue Scientifique et Technique de la Defense-40 Trimestre 1991, pp. 11-15.

15. F.E. Walker and R.J. Wasley, Combust. and Flame 22, 53 (1974).

16. R.R. McGuire and D.L. Ornellas, Propell, and Explo. 4, 23 (1979).

17. D.W. Brenner, F.E. Walker, et al, Int. J. of Quantum Chem., Quantum Chemistry Symposium 23, 1989. John Wiley and Sons, Inc. (1989)

18. A.M. Karo and J.R. Hardy, Fast Reactions in Energetic Systems, pp. 61i-643, C.Capellos and R.F. Walker (editors) D. Reidel Publishing Co. (1981) NATO Advanced Study Institute, Series C: Mathematics and Physical Sciences.

19. D.H. Robertson, D.W. Brenner, et al, Shock Compression of Condensed Matter 1991, S.C. Schmidt, R.D. Dick, J.W. Forbes, D.G. Tasker (editors) 1992, Elsevier Science Publishers B.V., pp. 123-126 (1992). 
20. D.W. Brenner, D.H. Robertson, et al, Microscopic Simulations of Complex Hydrodynamic Phenomena, Edited by $M$. Mareschal and B.I. Holian, Plenum Press, New York, pp.111-123 (1992).

21. M. Peyrard, S. Odiot, et al, J. Appl. Phys. 57, 2626 (1985).

22. R.A. Graham, J. Phys. Chem. 83, 3048 (1979).

23. A.N. Dremin and O.N. Breusov, Priroda 12, 10 (1971).

24. B.W. Dodson, E.I. Venturini and J.E. Rogers, Proceedings of the 4th Annual Topical Conf. on Shock Waves in Condensed Matter, Spokane, WA 1985 , edited by Y.M. Gupta, PIenum Press, New York, 1986.

25. F.J. Owens anid J. Sharma, J. Appl. Phys. 51, 1494 (1980).

26. R. Lewin, A New Window Onto the Chemists' Big Bang, science 238, 1512 (1987).

27. N.F. Scherer, A.H. Zewail, et al, J. Chem. Phys. 87, 2395 (1987).

28. M. Dantus, M.J. Rosen and A.H. Zewail, J. Chem. Phys. 87, 2395 (1987).

29. F.E. Walker, Propellants and Explosives 15, 157 (1990).

30. M.J. Kamlet and S.J. Jacobs, J. Chem. Phys. 48, 23 (1968).

31. K. Tanaka, S. Oinuma, et al, Shock Compression of Condensed Matter 1989, S.C. Schmidt, J.N. Johnson, L.W. Davison (editors) Elsevier Science Publishers B.V., 1990.

32. J.I. Austing and A.J. Tulis, Proceedings of the 14 th Int. Pyrotechnics Seminar, Jersey, Channel Islands, UK, 18-22 September 1989, pp. 583-601.

33. J.L. Austing and A.J. Tulis, et al, Proceedings of the 16 th Int. Pyrotechnics Seminar, Jönköping, Sweden, 24-28 June 1991 , pp. 274-288.

34. F.E. Walker and R.J. Wasley, Explosivstoffe 17, 9 (1969).

35. A.N. Dremin and V.Yu, Klimenko, Progress in Aeronautics and Astronautics, edited by J.R. Bowen, N. Manson, A.K. Oppenheim and R.I. Soloukhin, Vol. 75, 253 (1981) New York.

36. F.E. Walker and R.J. Wasley, et al, Lawrence Iivermore National Laboratory, CA Report No. UCRL-75339, 1974.

37. F.E. Walker, Propel1., Explo., Pyrotech. 7, 2 (1982).

38. J.J. Gilman, Shock Compression of Condensed Matter 1989 S.C. Schmidt, J.N. Johnson and L.W. Davison (editors) Elsevier Science Publishers B.V., p. 267 (1990). 
Questions - Answers, Comments

Shackelford - Dlott :

Q : Take for example, a normal molecule like HMX and a deuterated molecule like HMX-d8. How might this deuterium substitution affect this model in terms of chemical reaction initiation, especially in terms of energy transfer rates and time to chemical reaction initiation?

A : Experiments by Dlott, Califano, Hochstrasser and Chronister show molecular energy transfer is faster in deuterated non-energetic molecules. In our model this reduces the possibility of hot-spot formation in up-pumping zone.

Califano : a comment I like very much the idea of hot spots presented by Dana. I would like to suggest that a hot spot is not only a trap for energy but also a center of scattering processes which can open new channels to the up conversion process.

\section{Nelson : a comment to Dremin}

You are correct that femtosecond spectroscopy of real shocked materials will be impossible if it requires synchronization of light pulses with shock loading at femtosecond accuracy. But other methods are possible.

For example, Yogi Gupta has suggested femtosecond pump-probe spectroscopy of a material with a shock wave passing through it. For example there is a narrow zone of endothermic reaction products they could be detected and characterized.

Dana Dlott has taken a different approach, building very small spatial structures into the sample to permit a kind of synchronization which could be done on subpicosecond time scales

\section{Delpuech - Dlott : a comment :}

A complement about a remark of Dr Dlott. The value of the temperature considerated in the proposition of excited state is not an average value.

Is the value that we can obtain, at the molecular scale, in the crossing under energy loading, of dislocations in the crystal? In this case this value is compatible with few electron-volts.

Of course at the begining we have excited states only in localised zones and not in the bulk of the explosive.

The question is not how the phonons give the energy of the shock at the molecule, but how an excited molecule gives with the phonon its energy to the other molecules in order to obtain a cooperative mode of decomposition.

\section{Boris - Dlott :}

$Q$ : What are the effects of energy transport from the sea of excited phonons into the hot spot ? Can this focusing of energy enhance the sensitivity enough to account for observed explosive behavior? 
A : Mechanical energy must flow to the hot spots in order for them to overheat. In unpublished work by us, we found the transport of energy to a hot spot by acoustic phonons did not have a substantial effect, although this work is not complete. One interesting effect we have discussed in our J.Chem.Phys. article involved hot spots formed in small grains of material. The (extensive) heat capacity of small grains is limited, so hot spots cannot become as hot. This led to our prediction of size effects in defect-assisted shock-induced chemistry.

\section{Odiot - Dlott :}

$\mathbf{Q}:$ I should agree with your model if you could explain to me how a shock may excite phonons through Gruneisen parameter in such a non equilibrium state of a shocked material.

A : The Grüneisen parameter $\Gamma=\Sigma_{\mathrm{i}} \gamma_{\mathrm{i}} \mathrm{c}_{\mathrm{i}} / \Sigma_{\mathrm{i}} \mathrm{c}_{\mathrm{i}}$ where $\gamma_{\mathrm{i}}$ are mode grüneisen parameters and $\mathrm{c}_{\mathrm{i}}$ are mode heat capacities. Upon a change in volume, the change of a mode's internal energy $\mathrm{E}_{\mathrm{i}}$ is proportional to $\gamma_{i}=-\mathrm{d} \ln \mathrm{E}_{\mathrm{i}} / \mathrm{d} \ln \mathrm{v}$. For phonons, $\gamma_{\mathrm{i}}$ is typically $10^{2}$ bigger than for vibrations. Thus the initial transfer of energy from a shock is principally to the phonons.

\section{Ramsay - Dlott :}

$\mathbf{Q}$ : Can your model of phonon pumping around a discontinuity (bubble) be compared with the data available in the pictures presented by Dr Presles on monday?

A : I don't know.

\section{Rullière - Dlott :}

$\mathbf{Q}$ : You showed multi steps absorption of phonons to reach vibrational excited state. Are the lifetimes of involved vibration and the probability to meet a phonon compatible to get a high probability for this process to occur efficiently?

A : In diatomic molecules, energy transfer from phonons to vibrations (multiphonon up-pumping) is quite inefficient. It involves a high-order anharmonic process with simultaneous absorption of $\mathrm{n}$-phonons (e.g. $\mathrm{n}>20$ for $\mathrm{N}_{2}$ ). In large molecules, up-pumping involves a lower-order process where $n=3-4$. This lower-order process is much more efficient. Up-pumping occurs by a sequence of many of these lower order steps. For example, it is possible for a larger molecule to absorb thousands of $\mathrm{cm}^{-1}$ on a $100 \mathrm{ps}$ scale.

Walker to workshop on what a shock really is, a comment :

Let's no forget what a detonation really is. As Prof Eyring explained many years ago, it is a momentum transfer process $-m_{1} v_{1}=m_{2} v_{2}$. It is the momentum of one layer of atoms accelerating and displacing the next layer - in a simplified view. The detonation velocity in HMX is $9.11 \AA$ in $10^{-13} \mathrm{~s}$. This means that the detonation front on the atomic scale is crossing the original position of each layer of atoms in $10^{-14} \mathrm{~s}$. Any chemical energy that would be released in $10 \mathrm{ps}$ would be 1000 layers of atoms behind the front with no understandable way of catching up to the 
front. In a $\mu \mathrm{s}$, it would be 100,000 layers too late. Energy strong enough to drive the detonation front must be available very near the front.

The ingenious spectroscopic instruments and devices discussed today would certainly be useful in studying shock initiation, but a laser beam burning a spot in an explosive sample or even making an impacting shock of a high velocity is not forming a detonation.

My concept of the extremly high kinetic energy from the extremly high momentum transfer producing enough force to fracture covalent bonds or cause very high velocity impacts on an atomic and molecular level to ensure chemical reaction and energy release within a time of $10^{-14}$ to $10^{-12}$ seems to be required. The kinetic energy in the detonation front is in the level of several ev.

Dlott to workshop in general about Walker's presentation. Comment

Dr Walker considers very intense shock waves characteristic of detonation (e.g. $40 \mathrm{GPa}$ ). In this regime, the kinetic energy of atoms is much greater than the energy of all covalent bonds. In this regime, his suggestion of efficient bond scission at the front seems reasonable.

In our model, we consider chemistry induced by weak shock waves. Then chemistry is not likely at the front but instead less efficient thermochemical bond cleavage will occur farther behind the front. Keep in mind these two models describe different situations. 


\section{SHOCK RESPONSE OF CONDENSED EMS - EXPERIMENTS}

Chairman : Pr Boris Kondrikov, Mendeleev University of Chemical Technology

Mesdames et Messieurs, Ladies and Gentlemen

I appreciate very much all the organizers of this exclusively important meeting for the opportunity to visit France, and in the first time in my life to have honor to be a Chairman of a session of one of the international Conference I was not be able to take part in during more than two decades.

The first day of our workshop was devoted mostly to the macroscopic aspects of the detonation processes. It was absolutely necessary to begin our discussion namely with these macroscopic approaches which were elaborated during about a century, having in mind the great experience accumulated at these investigations is a ground for all the future understanding of thin structure of matter. Now we have to discuss some of them, I would say probably the best of them. It was the wonderful lecture here given by Dr.K.Nelson on femtosecond measurements we had possibility to hear just now, and the reports of Dr.Y.Gupta and Dr.Dlott on pico-and nano-second measurements that should be presented in this session.

I would like to note that I had opportunity to discuss all three of the reports this year on Gordon Conference New Hampshire USA, and Dr.Gupta's paper also on Zel'dovich Conference in Moscow. I believe it is a very good idea to present them in different meetings and for the broad audience, because all of these works are absolutely new word in field of shock and detonation transformations, and correspondingly they need much attention and one could say the deep penetration into the essence of the new results obtained in the course of the very hard work (as well as the very big expenditures). In this connection I would like to mention here about a role of Office Naval Research and personally Dr.R.Miller, who have partly supported these programs, having in mind first of all the obvious necessity of these investigations for the fundamental science of developments as a natural base for all the future practical applications. I have to note also that though strickly speaking not all of the data obtained are concerned literally with behavior of high explosives at very strong shock stress during the very short period of time, the field of science we have been penetrating into at these investigations is so much more complicated than any other, in this part of physics and chemistry, that we need to use all the possible ways to reach the positive and definitive results.

It should be also taken into account that as a matter of fact we have now instead of the single classical theory of detonation the many kinds of detonation-alike processes for solid and liquid 
compounds, for composite explosives (some potentially very interesting lectures will be given later), at relatively low, and at very high pressures and temperatures. Everyone of the processes needs special examination and employment of all possible means to expose the essence of them, and to use it in science and technology.

Recent Developments to understand Molecular Changes in Shocked Energetic Materials ; D.D. Dlott

Picosecond Dynamics behind the Shock Front ; Y.M. Gupta 